\title{
Gain scheduled integral linear quadratic control for quadcopter
}

\author{
J Shah, M Okasha*, W Faris \\ Department of Mechanical Engineering, International Islamic University Malaysia, Kuala Lumpur, Malaysia \\ *Corresponding author E-mail: mokasha@iium.edu.my
}

\begin{abstract}
The findings of this paper are focused on the dynamics and control of a quadcopter using a modified version of a Linear Quadratic Regulator (LQR) control approach. The classical LQR control approach is extended to include an integral term to improve the quad copter tracking performance. The mathematical model is derived using the Newton-Euler method for the nonlinear six DOF model that includes the aerodynamics and detailed gyroscopic moments as a part of the system identification process. The linearized model is obtained and it is characterized by the heading angle (yaw angle) of the quadcopter. The adopted control approach is utilizing the LQR method to track several trajectories i.e. helical and lissajous curve with significant variation in the yaw angle. The integral term is introduced to the controller in order to minimize the steady state errors observed. The controller is modified to overcome difficulties related to the continuous changes in the operation points and to eliminate the chattering that was observed in the control technique. Numerical non-linear simulations are performed using MATLAB \& Simulink to illustrate to accuracy and effectiveness of the proposed controller.
\end{abstract}

Keywords: quadcopter; LQR; gain scheduled; dynamics and control.

\section{Introduction}

Over the years, the quadcopter has caught the attention of many researchers due to their resilience, versatility, compact size and their vast applications in various fields. There is a potential widespread use of these vehicles in the civil sector such as inspection of terrain, pipelines and buildings, firefighting, aerial photography, search and rescue, mapping, remote sensing, etc., as well for intelligence, reconnaissance and surveillance in the military sector [1]. It is a very highly non-linear system that has six degrees of freedom (DOM) but has only four actuators to control the outputs, which makes it an under actuated system by a degree of two. Moreover, the design of a controller is also subjected to the consideration of controller saturation where the motor dynamics have to be carefully designed in order to achieve practical control effort from the rotors when implemented to the physical hardware [2].

Researchers have been working to improve the performance of the quadcopters, especially when it comes to the response and stability of the system. Thus, researchers have designed various control techniques like Proportional-Integral-Derivative (PID), Linear Quadratic Regulator (LQR), Sliding Mode Control (SMC), Model Predictive Control (MPC) and Back-stepping to control the quadcopter [3-10]. The controllers designed are linear in nature, therefore when implemented on the actual non-linear system, the controller reflects dissimilar effects on the system. Moreover, external, unaccounted for, disturbances and other inherent system limitations also propagate undesired performance of the system in real-time applications.

The LQR control is one of the conventional non-linear control techniques that is widely used in a range of control system applications. In many literatures, it has been compared to other conventional control approaches like PID control or fuzzy control. The implementation of the LQR control technique in an inverted pen- dulum system produced better performance results with acceptable overshoot and undershoot and a faster response when compared to the PID controller's performance [11]. Even though limited in linear systems, the LQR method is very useful when non-linear systems are linearized, especially multivariable systems, about defined equilibrium points. An example of that is an indoor micro quadrotor that was subjected to the performance comparison of a PID and LQR control where the results of the LQ control technique were favourable [12].

This study aims on improving the design of a gain scheduled LQR controller by adding an integral term that minimizes the steady state error of the output. Detailed mathematical model is formulated using Newton-Euler method for the quadcopter, taking in account the gyroscopic rotor dynamics and also aerodynamic drag and moment terms, whereas the actuator saturation and chattering of the control input signal is considered during the design of the controller.

\section{Dynamic model}

It is imperative to establish a coordinate system and develop mathematical model using the Newton-Euler equations of motion that reflect the dynamics of the system. Thus, system identification prior to modelling helps in obtaining an accurate model. A number of physical parameters such as mass, moment of inertia and arm length are to be determined based on the configuration of the quadcopter. In this case, the model is developed for an X configuration quadcopter including inertial moments $\left(I_{x}, I_{y}, I_{z}\right)$, aerodynamic translational $\left(K_{t,}, K_{t y}, K_{t z}\right)$ and rotational $\left(K_{r y}, K_{r y}, K_{r z}\right)$ drag constants, gravitational constant $g$, mass of the quadcopter $m$, moment arm $l$, angular velocity of the body $(p, q, r)$, Euler angles $(\varphi, \theta, \psi)$, rotor's inertia $I_{r}$, rotor's speed $\omega_{r}$, thrust force $F$ and torque produced by the rotors $T$. The system coordinates of the quadrotor is illustrated in Figure 1. 


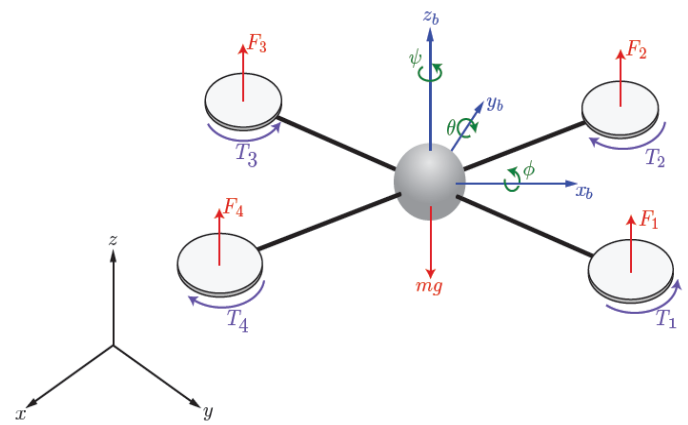

Figure 1: System coordinates of quadcopter [10]

The equations of motions are derived through Newton-Euler approach which are shown in Eqn. 1 to Eqn. 9, where $(x, y, z)$, $(\dot{x}, \dot{y}, \dot{z})$ and $(\ddot{x}, \ddot{y}, \ddot{z})$ are components of position, velocity and acceleration vectors in the world coordinate frame, $(s, c, t)$ denote $\sin$, cosine and tangent trigonometric function and $\left(u_{1}, u_{2}, u_{3}, u_{4}\right)$ are the components of the control input vector defined by Eqn. 10 to Eqn. 13, respectively.

$\ddot{x}=-\left(K_{t_{x}} \dot{x}-m u_{1}(s \varphi s \psi+c \varphi s \theta c \psi)\right) / m$

$\ddot{y}=-\left(K_{t_{y}} \dot{y}+m u_{1}(s \varphi c \psi-c \varphi s \theta s \psi)\right) / m$

$\ddot{z}=-\left(g m+K_{t_{z}} \dot{z}-m u_{1} c \theta c \varphi\right) / m$

$\dot{\varphi}=p+r c \varphi t \theta+q s \varphi t \theta$

$\dot{\theta}=q c \varphi-r s \varphi$

$\dot{\psi}=r c \varphi / c \theta+q s \varphi / c \theta$

$\dot{p}=-\frac{K_{r_{x}} p-I_{x} u_{2}-I_{y} q r+I_{z} q r+I_{r} q \omega_{r}}{I_{x}}$

$\dot{q}=\left(-K_{r_{y}} q+I_{y} u_{3}-I_{x} p r+I_{z} p r+I_{r} p \omega_{r}\right) / I_{y}$

$\dot{r}=\frac{-K_{r_{z}} r+I_{z} u_{4}+I_{x} p q-I_{y} p q}{I_{z}}$

$u_{1}=\frac{F_{1}+F_{2}+F_{3}+F_{4}}{m}$

$u_{2}=\frac{\left(-F_{1}+F_{2}+F_{3}-F_{4}\right) l}{I_{x}}$

$u_{3}=\frac{\left(-F_{1}-F_{2}+F_{3}+F_{4}\right) l}{I_{y}}$

$u_{4}=\left(T_{1}-T_{2}+T_{3}-T_{4}\right) / I_{z}$

From Eqn. 1 to Eqn. 9, it can be clearly seen that the system is under actuated as it has four inputs $\left(u_{1}, u_{2}, u_{3}, u_{4}\right)$ and six coordinate outputs $(x, y, z, \varphi, \theta, \psi)$

\section{3. $L Q R$ controller design}

Eqn. 14 shows the compact form of the nonlinear dynamical model, where $\mathbf{x}$ is the input vector and $\mathbf{u}$ is the control input vector of the quadcopter. These vectors are defined as in Eqn. 15 and Eqn. 16.

$\dot{\mathbf{x}}=\mathbf{f}(\mathbf{x}, \mathbf{u})$

$\mathbf{x}=(x, y, z, \dot{x}, \dot{y}, \dot{z}, \varphi, \theta, \psi, p, q, r)$

$\mathbf{u}=\left(u_{1}, u_{2}, u_{3}, u_{4}\right)$

The linearization of the model at equilibrium produces Eqn. 17. Eqn. 18 and Eqn. 19 are resulted from solving Equation 17.

$\mathbf{0}=\mathbf{f}\left(\mathbf{x}_{s S}, \mathbf{u}_{s s}\right)$

$\mathbf{x}_{s S}=\left(x_{s S}, y_{s s}, z_{s S}, 0,0,0,0,0, \psi_{s S}, 0,0,0\right)$

$\mathbf{u}_{s s}=(g, 0,0,0)$

The linearized model is obtained around the steady states values to design the LQR controller as can be seen in Eq. 20, where $\delta \mathbf{x}=\mathbf{x}-\mathbf{x}_{\mathrm{sS}}$ and $\delta \mathbf{u}=\mathbf{u}-\mathbf{u}_{s s}$ while Jacobian matrix $\mathbf{A}$ and $\mathbf{B}$ are given by Eqn. 21 and Eqn. 22, respectively.

$\delta \dot{\mathbf{x}}=\mathbf{A} \delta \mathbf{x}+\mathbf{B} \delta \mathbf{u}$

$\mathbf{B}=\frac{\partial \mathbf{f}}{\partial \mathbf{u}_{\left(\mathbf{x}_{s s}, \mathbf{u}_{s s}\right)}}=\left[\begin{array}{cccc}0 & 0 & 0 & 0 \\ 0 & 0 & 0 & 0 \\ 0 & 0 & 0 & 0 \\ 0 & 0 & 0 & 0 \\ 0 & 0 & 0 & 0 \\ 1 & 0 & 0 & 0 \\ 0 & 0 & 0 & 0 \\ 0 & 0 & 0 & 0 \\ 0 & 0 & 0 & 0 \\ 0 & 1 & 0 & 0 \\ 0 & 0 & 1 & 0 \\ 0 & 0 & 0 & 1\end{array}\right]$

$\mathbf{A}=\frac{\partial \mathbf{f}}{\partial \mathbf{x}_{\left(\mathbf{x}_{s s}, \mathbf{u}_{s s}\right)}}$

$\left[\begin{array}{cccccccccccc}0 & 0 & 0 & 1 & 0 & 0 & 0 & 0 & 0 & 0 & 0 & 0 \\ 0 & 0 & 0 & 0 & 1 & 0 & 0 & 0 & 0 & 0 & 0 & 0 \\ 0 & 0 & 0 & 0 & 0 & 1 & 0 & 0 & 0 & 0 & 0 & 0 \\ 0 & 0 & 0 & -\frac{K_{t_{x}}}{m} & 0 & 0 & g s \psi & g c \psi & 0 & 0 & 0 & 0 \\ 0 & 0 & 0 & 0 & -\frac{K_{t_{y}}}{m} & 0 & -g c \psi & g s \psi & 0 & 0 & 0 & 0 \\ 0 & 0 & 0 & 0 & 0 & -\frac{K_{t_{z}}}{m} & 0 & 0 & 0 & 0 & 0 & 0 \\ 0 & 0 & 0 & 0 & 0 & 0 & 0 & 0 & 0 & 1 & 0 & 0 \\ 0 & 0 & 0 & 0 & 0 & 0 & 0 & 0 & 0 & 0 & 1 & 0 \\ 0 & 0 & 0 & 0 & 0 & 0 & 0 & 0 & 0 & 0 & 0 & 1 \\ 0 & 0 & 0 & 0 & 0 & 0 & 0 & 0 & 0 & -\frac{K_{r_{x}}}{I_{x}} & 0 & 0 \\ 0 & 0 & 0 & 0 & 0 & 0 & 0 & 0 & 0 & 0 & -\frac{K_{r_{y}}}{I_{y}} & 0 \\ 0 & 0 & 0 & 0 & 0 & 0 & 0 & 0 & 0 & 0 & 0 & -\frac{K_{r_{z}}}{I_{z}}\end{array}\right]$

As found in Ref. [10], the system has a rank of 12, which makes it observable and controllable with output feedback $\mathbf{y}=(x, y, z, \psi)$. The control input vector can be computed using LQR controller as in Eqn. 23 where $\mathbf{u}=\delta \mathbf{u}+\mathbf{u}_{s s}$. The gain matrix $\mathbf{K}$ that is used to minimize cost function in Eqn. 24 can be computed using the following Matlab command $\mathbf{K}=\operatorname{lq} \mathbf{r}(\mathbf{A}, \mathbf{B}, \mathbf{Q}, \mathbf{R})$. In Eqn. $24, \mathbf{Q}$ is the 
symmetric positive definite weighting matrices for the system state and $\mathbf{R}$ is the symmetric positive definite weighting matrices for the control input.

$\delta \mathbf{u}=-\mathbf{K} \delta \mathbf{x}$

$\mathrm{J}=\int_{0}^{\infty}\left(\delta \mathbf{x}^{T} \mathbf{Q} \delta \mathbf{x}+\delta \mathbf{u}^{T} \mathbf{R} \delta \mathbf{u}\right) \mathrm{dt}$

The designed controller is tested by tracking a time varying helical trajectory with varying heading angle $\psi$. The controller switch for the gain scheduling is described in Eqn. 25, where the gain is recomputed after every 30 degrees. The controller is also used with a continuous gain in which the gain changes as a function of the heading angle.

$$
\mathbf{u}(\mathbf{x})=\left\{\begin{aligned}
\mathbf{u}_{1}\left(\mathbf{x} ; \frac{\pi}{12}\right), & 0 \leq \psi(t) \leq \frac{\pi}{6} \\
\mathbf{u}_{2}\left(\mathbf{x} ; \frac{\pi}{4}\right), & \frac{\pi}{12} \leq \psi(t) \leq \frac{\pi}{3} \\
& \vdots \\
\mathbf{u}_{12}\left(\mathbf{x} ; \frac{23 \pi}{4}\right), & \frac{11 \pi}{6} \leq \psi(t) \leq 2 \pi
\end{aligned}\right.
$$

The integral term is introduced to the controller as shown in Eqn. 26 , where $\boldsymbol{\sigma}$ is the derivative of the error term between the output of the tracking states and their reference value, and $y$ is the subset of the measureable state vector $x$ and has the same dimension as the control vector $u$.

$\dot{\boldsymbol{\sigma}}=y-r$

As $y=C x$, Eqn. 26 can also be represented as Eqn. 27.

$\dot{\boldsymbol{\sigma}}=C x-r$

Block diagram of the gain-scheduled integral control is shown in Figure 2. The new control law in definition of the optimal gain values of the states $K_{I}$ and the optimal integral values $K_{2}$ is defined in Eqn. 28. The gain matrix $\boldsymbol{K}_{\boldsymbol{i}}$ can be computed using the following Matlab command $\boldsymbol{K}_{\boldsymbol{i}}=\operatorname{lqi}(\mathbf{S Y S}, \mathbf{Q}, \mathbf{R})$, where $K_{l}$ is the optimal gain values for the weighting matrix of the states $\boldsymbol{Q}_{x}$ and $K_{2}$ is the optimal gain values for the weighting matrix of integral tracking states $\boldsymbol{Q}_{\boldsymbol{\delta}}$. This comes from the augmented state vector given as Eqn. 30 .

$\boldsymbol{K}_{\boldsymbol{i}}=\left(\begin{array}{ll}K_{1} & K_{2}\end{array}\right)$

$\boldsymbol{u}=-K_{1}\left(\boldsymbol{x}-\boldsymbol{x}_{\boldsymbol{s} s}\right)-K_{2} \boldsymbol{\sigma}$

$\delta=\left(\begin{array}{ll}x & \sigma\end{array}\right)^{T}$

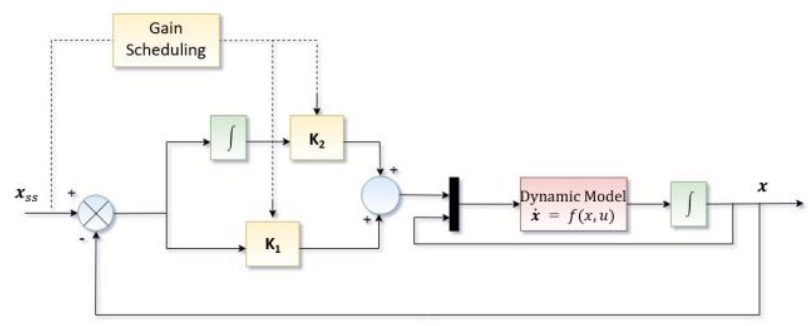

Fig. 2: Block diagram of gain-scheduled integral control

The cost function can be written as follow:

$\mathrm{J}=\int_{0}^{\infty}\left(\delta^{T} \mathbf{Q}_{\delta} \delta+\mathbf{u}^{T} \mathbf{R u}\right) \mathrm{dt}$
$\mathbf{Q}_{\delta}=\left(\begin{array}{cc}\mathrm{Q}_{x} & 0 \\ 0 & \mathrm{Q}_{\sigma}\end{array}\right)$

$\mathrm{J}=\int_{0}^{\infty}\left(\delta \mathbf{x}^{T} \mathbf{Q}_{x} \delta \mathbf{x}+\delta \boldsymbol{\sigma}^{T} \mathbf{Q}_{\boldsymbol{\sigma}} \delta \boldsymbol{\sigma}+\delta \mathbf{u}^{T} \mathbf{R} \delta \mathbf{u}\right) \mathrm{dt}$

\section{Results and discussions}

The performance of the controller is assessed through simulations using MATLAB and Simulink environment. The parameters used in the simulation are taken from Ref. [10], considering the actuators' saturation limits. The $\mathbf{Q}$ and $\mathbf{R}$ matrices are computed manually by trial and error tuning method. These matrices are diagonal and have the following values: $\mathbf{Q}=\operatorname{diag}(10,10,10,1,1,1,1,1$, $10,1,1,1)$ and $\mathbf{R}=\operatorname{diag}(0.001,0.001,0.001,0.001)$ while for the weighting matrix for integral part, the numerical value is $\mathbf{Q}_{\boldsymbol{\sigma}}=$ $\operatorname{diag}(0.001,0.001,0.1,0.1)$.

The simulation results presented on the proposed controller are based on the following cases: (1) tracking of helical trajectory using gain-scheduled LQR control, (2) tracking of helical and lissajous trajectory with using gain-scheduled integral LQR control and (3) controller input for the scheduled gain and the continuous gain. In the simulations, the red dotted lines represent the planned trajectory whereas the blue lines represent the actual trajectory as a result of the designed controller. Figure 3 and Figure 4 show the effect of gain scheduled integral LQR control on a helical trajectory. Figure 5 and Figure 6 show the same effect using a lissajous curve trajectory. The lissajous curve is parametrized by Eqn. 34 and Eqn. 35. For this simulation, $A=10, B=5, a=2, b=$ 3 and $\omega=0$ are used.

$$
x=A \sin (a t+\omega)
$$

$y=B \sin (b t)$
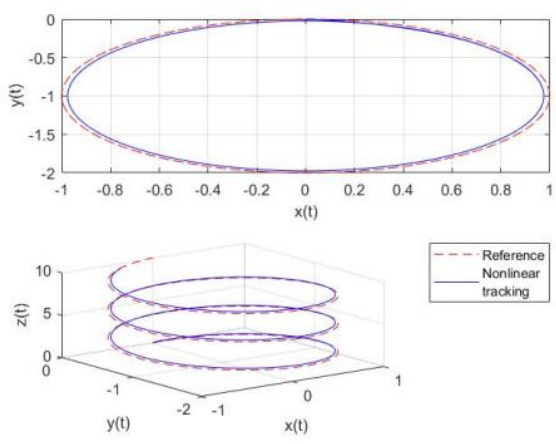

(a)
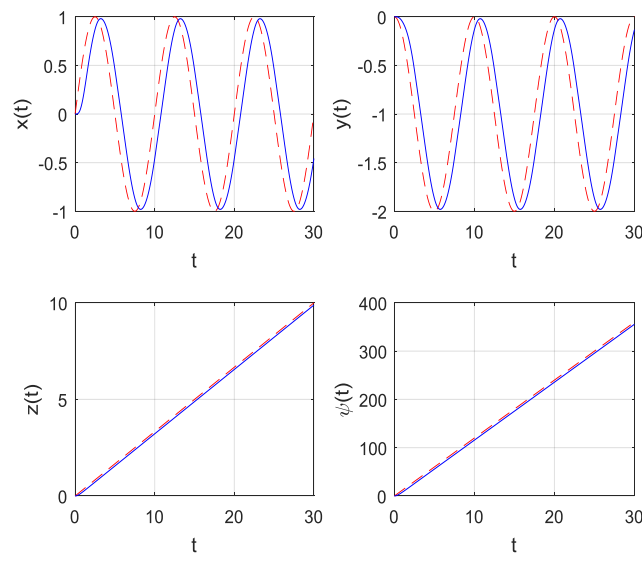

(b) 
Fig. 3: Gain Scheduled LQR: (a) Helical trajectory tracking in 2D and 3D (b) Tracking of states
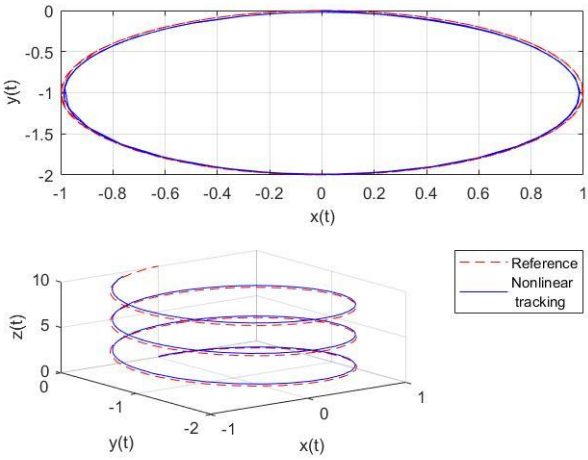

Tracking

(a)
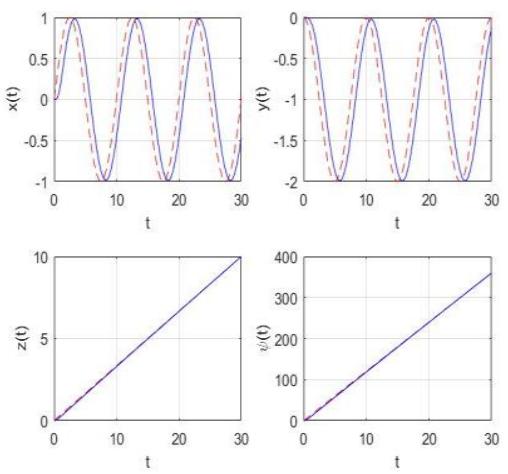

(b)

Fig. 4: Gain Scheduled Integral LQR: (a) Helical trajectory tracking in 2D and 3D (b) Tracking of states
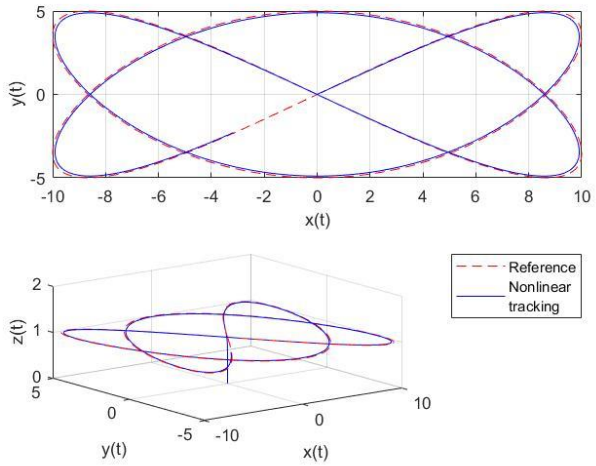

(a)
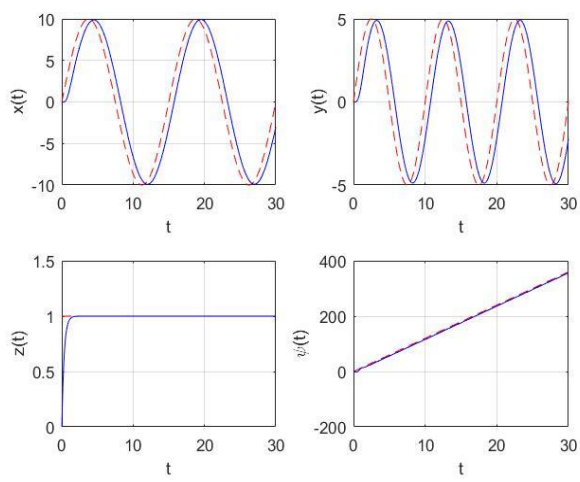

(b)

Fig. 5: Gain Scheduled LQR: (a) Lissajous trajectory tracking in 2D and 3D (b) Tracking of states
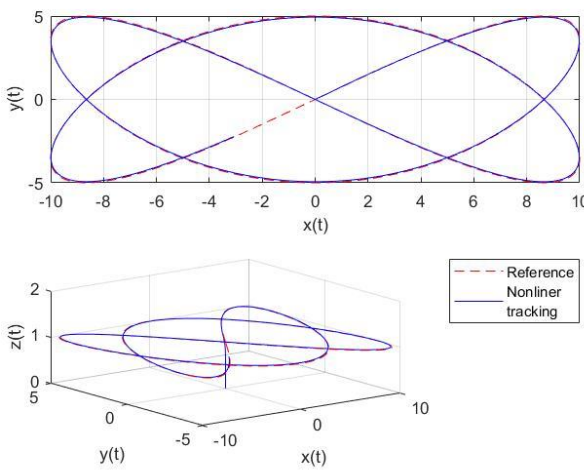

(a)
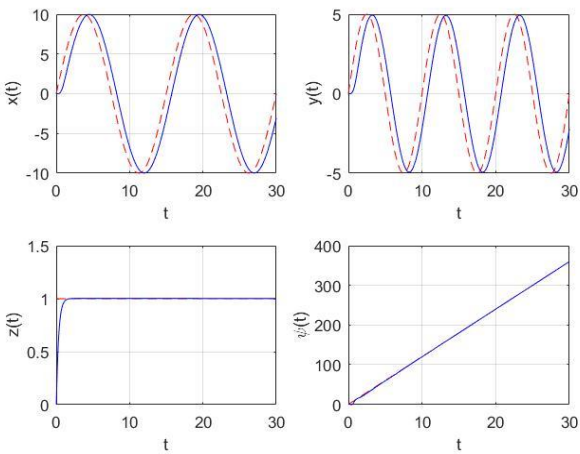

(b)

Fig. 6: Gain Scheduled Integral LQR: (a) Lissajous trajectory tracking in $2 \mathrm{D}$ and $3 \mathrm{D}(\mathrm{b})$ Tracking of states

As calculated, the percentage decrease in the steady state error in tracking the heading angle $\boldsymbol{\psi}$ of helical trajectory is $95.6 \%(4.3854$ to 0.222$)$ and $56.5 \%(0.0865$ to 0.0376$)$ that of the lissajous curve. For tracking state $\boldsymbol{z}$, a percentage decrease of $94.8 \%(0.1155$ to $0.0059)$ is observed for the helical trajectory, whereas a minor increase in steady state error of $4 \%(0.00$ to 0.04$)$ is seen for the lissajous curve. It is observed that these states were largely subjected to minimization of the steady state error whereas the $\boldsymbol{x}$ and $\boldsymbol{y}$ states, due to the coupling, depicted very less change seen in Figures 4(b) and 6(b). Furthermore, the limitations of the control efforts need to be considered during design due to physical implementation. Figure 7(a) shows the control efforts to track the helical trajectory for the gain-scheduled case. Due to switching discretely between operating points, the control input signals show a form of discontinuity and chattering which can affect the physical implementation of the control law. Figure 7(b) illustrates the elimination of chattering by the employment of a continuous gain.
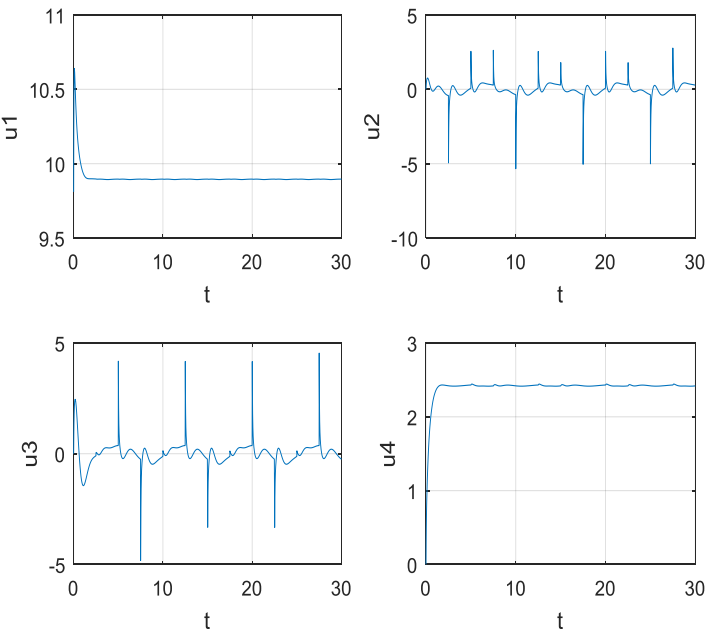

(a) 

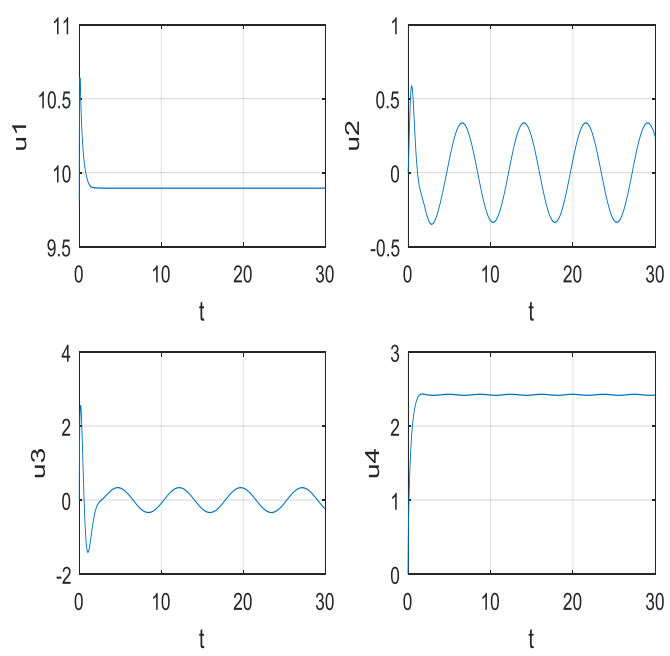

(b)

Fig. 7: Control input for the helical trajectory: (a) gain-scheduled, (b) continuous gain

\section{Conclusion}

To summarize, quadcopters have been introduced and the detailed mathematical model for them has been derived, which includes the aerodynamic drag and gyroscopic moment terms that are often ignored in various literatures by using the Newton-Euler approach. Then, a parameter varying linearized model is obtained by linearizing the nonlinear model around the steady state values. The LQR control approach is designed that uses gain scheduling as a function of the heading angle $(\psi)$ in order to eliminate the limitation in tracking caused by linearization about an operating point. The tolerance of the heading angle was set as $\psi_{t o l}=\pi / 6$ that would render the gain values over multiple operating points for the tracking signal. Furthermore, an integral term has been included to the proposed gain-scheduled controller. It is observed that the tracking issue is resolved with minimized steady state error in the tracking signals. The continuous gain control law is able to overcome chattering and discontinuity problems of control input signals as observed in the gain scheduled controller. To improve the tracking, the model can be designed such that the steady state error of the tracking signals $x$ and $y$ are also minimized. The designed controller can be tested and implemented on a quadcopter hardware in the loop real time simulation, to observe and assess the performance of the designed controller in the real world.

\section{References}

[1] Nanomi K, Kendoul F, Suzuki S, Wang W \& Nakazawa D (2010), Autonomous Flying Robots: Unmanned Aerial Vehicles and Micro Aerial Vehicles. Springer

[2] Hoffmann GM, Huang H, Waslander SL \& Tomlin CJ (2007), Quadrotor helicopter flight dynamics: theory and experiment. AIAA Guidance, Navigation and Control Conference

[3] Okasha M, Shah J, Fauzi W \& Hanouf Z (2017), Gain scheduled linear quadratic control of quadcopter. IOP Conference Series: Materials Science and Engineering 270, 012009

[4] Bouabdallah S, Noth A \& Siegwart R (2004), PID vs LQ control techniques applied to an indoor micro quadrotor. IEEE/RSJ International Conference on Intelligent Robots and Systems

[5] Li J \& Li Y (2011), Dynamic analysis and PID control for a quadrotor. International Conference in Mechatronics and Automation

[6] Bouabdallah S \& Siegwart R (2005), Backstepping and slidingmode techniques applied to an indoor micro quadrotor. IEEE International Conference in Robotics and Automation

[7] Nicol C, Macnab CJB \& Ramirez-Serrano A (2011), Robust adaptive control of a quadrotor helicopter. Mechatronics 21, 927-938

[8] Mueller WM \& D'Andrea R (2013), A model predictive controller for quadrocopter state interception. European Control Conference
[9] Elkholy H (2014), Dynamic Modeling and Control of A Quadrotor using Linear and Nonlinear Approaches. Thesis, American University in Cairo

[10] Sawyer S (2015), Gain-Scheduled Control of a Quadcopter UAV. Thesis, University of Waterloo

[11] Nasir A, Ahmed M \& Rahmat M (2008), Performance comparison between LQR and PID controller for an inverted pendulum system. International Conference on Power Control and Optimization

[12] Bouabdallah S, Noth A \& Seigwart R (2014), PID vs LQ control technniques applied to an indoor micro quadrotor. Autonomous Systems Laboratory Swiss Federal Institute of Technology 